\title{
Age-related Differences in the Delivery of Cardiac Management to Women Versus Men With Acute Myocardial Infarction in Japan
}

\author{
Tokai Acute Myocardial Infarction Study: TAMIS \\ Yoshihisa HiRAKawa, ${ }^{1} \mathrm{PhD}$, Yuichiro Masuda, ${ }^{1} \mathrm{PhD}$, \\ Kazumasa UemurA, ${ }^{2}$ PhD, Masafumi KuZuYA, ${ }^{1}$ PhD, Takaya Kimata, ${ }^{1}$ MD, \\ and Akihisa IGUCHI, ${ }^{1} \mathrm{PhD}$
}

\begin{abstract}
SUMMARY
It is of concern that women are more likely to undergo fewer diagnostic tests and receive less treatment for acute myocardial infarction (AMI) than men. However, it is still unclear whether gender differences exist according to age groups. Therefore, we studied the influence of gender on the delivery of cardiac management according to two age groups $(<65, \geqq 65)$ in Japan. Data from the Tokai Acute Myocardial Infarction Study (TAMIS) sample were used. This is a retrospective study of all consecutive patients admitted to the 13 acute care hospitals in the Tokai region of Japan, which includes Aichi and Shizuoka Prefectures, with a diagnosis of AMI from 1995 to 1997. A total of 143 younger women, 822 younger men, 391 older women, and 611 older men were included. Information concerning patient demographics, in-hospital course, comorbid conditions, electrocardiography (ECG), ultrasound-echocardiography (UCG), treadmill test (TMT), coronary angiography (CAG), percutaneous transluminal coronary angioplasty (PTCA), coronary artery bypass graft (CABG), intra-aortic balloon pump (IABP), mechanical ventilation, and in-hospital or discharge medication (thrombolytics, vasopressors, aspirin, $\beta$ blockers, angiotensin-converting enzyme (ACE) inhibitors, calcium antagonists, nitrates) were collected. Among the young, after controlling for these baseline variables, women were significantly less likely to undergo PTCA compared to men (OR, 0.54, 95\% CI, 0.350.82). After controlling for these baseline variables, only lipid-lowering therapy tended to be more frequent in women than in men among the elderly (OR, 2.79, 95\% CI, 1.47-2.58). The findings suggest that younger women with AMI are less likely than younger men to undergo PTCA, and that older women with AMI are more likely to receive lipid-lowering therapy. (Int Heart J 2005; 46: 939-948)
\end{abstract}

Key words: Acute myocardial infarction (AMI), Gender, Age group, Percutaneous transluminal coronary angioplasty (PTCA), Lipid-lowering therapy

From the Departments of ${ }^{1}$ Geriatrics and ${ }^{2}$ Internal Medicine, Nagoya University Graduate School of Medicine, Aichi, Japan.

Address for correspondence: Yoshihisa Hirakawa, MD, Department of Geriatrics, Nagoya University Graduate School of Medicine, 65 Tsuruma-cho, Showa-ku, Nagoya, Aichi 466-8550, Japan.

Received for publication May 10, 2005.

Revised and accepted July 14, 2005. 
CARDIOVASCULAR disease is a leading cause of death throughout the world, including in Japan, where Western-style diets are being increasingly adopted., ${ }^{1,2}$ In Western countries, it is of concern that women are less likely to undergo acute myocardial infarction (AMI) diagnostic tests and treatments. ${ }^{1,3-5)}$ Some studies have shown this, while others have suggested that women receive similar degrees of diagnostic tests and treatments for AMI as men. ${ }^{1,2,4,6-10)}$ There is still a significant amount of controversy concerning this matter so it has been impossible to come to any definite conclusion. It remains unclear whether gender differences in these treatments exist. ${ }^{11,12)}$

In addition, it has been suggested that the elderly with AMI are treated less aggressively than younger persons. ${ }^{1,13,14)}$ Therefore, we studied whether gender differences in AMI management are different between younger and older populations, as previously suggested by Montague, et al. ${ }^{15)}$

The purpose of this multihospital retrospective study was to study age-specific gender differences in the use of medications and the utilization of diagnostic and revascularization procedures among patients hospitalized for AMI.

\section{Methods}

Study population: Data from the Tokai Acute Myocardial Infarction Study (TAMIS), a multihospital retrospective study in the Tokai region of Japan (Central Japan), were used. All of the study subjects were adult patients hospitalized due to a diagnosis of AMI at thirteen acute care hospitals between January 1995 and December 1997. Their diagnoses were confirmed by subsequent chart reviews. Patients admitted more than once with a diagnosis of AMI were included in the analysis. With regards to the recruitment of participant hospitals, we first selected the major hospitals which had an interchange of personnel with Nagoya University Hospital, where we are based. Second, we sent a prospectus about our research to the hospitals selected, 13 of which approved the study. The 13 hospitals were municipal or nonprofit general hospitals which were able to perform coronary angiography (CAG) and percutaneous coronary intervention (PTCA). Not all of the hospitals had a department of cardiovascular surgery or could conduct coronary artery bypass grafting (CABG) during the study period, however, we could not make identify the hospitals even by follow-up interview with the hospital staff in charge.

Data collection: We abstracted the baseline and procedural characteristics from detailed chart reviews which included both physician notes and nursing notes by physicians or skilled nurses educated to obtain medical records. The chart abstractors were blinded to the study hypothesis or anticipated study results. The questionnaire contained information on patient demographics, the in-hospital 
course (length of stay, ICU/CCU transfer), comorbid conditions, clinical presentation (body mass index, body temperature, systolic arterial pressure, heart rate, chest pain, pulmonary edema, shock, bleeding, Killip class, ultrasound-echocardiogram (UCG), treadmill test (TMT), locations of MIs, and earlier admission activity of daily living (ADL)), procedural characteristics [CAG, PTCA, CABG, intra-aortic balloon pump (IABP), mechanical ventilation, time from onset to angiography, and in-hospital and discharge medication (thrombolytics, vasopressors, aspirin, $\beta$-blockers, angiotensin-converting enzyme (ACE) inhibitors, calcium antagonists, nitrates)]. A history of various comorbid conditions was recorded as present if it was documented in the medical charts. If no information

Table I. Baseline Characteristics of the Patients

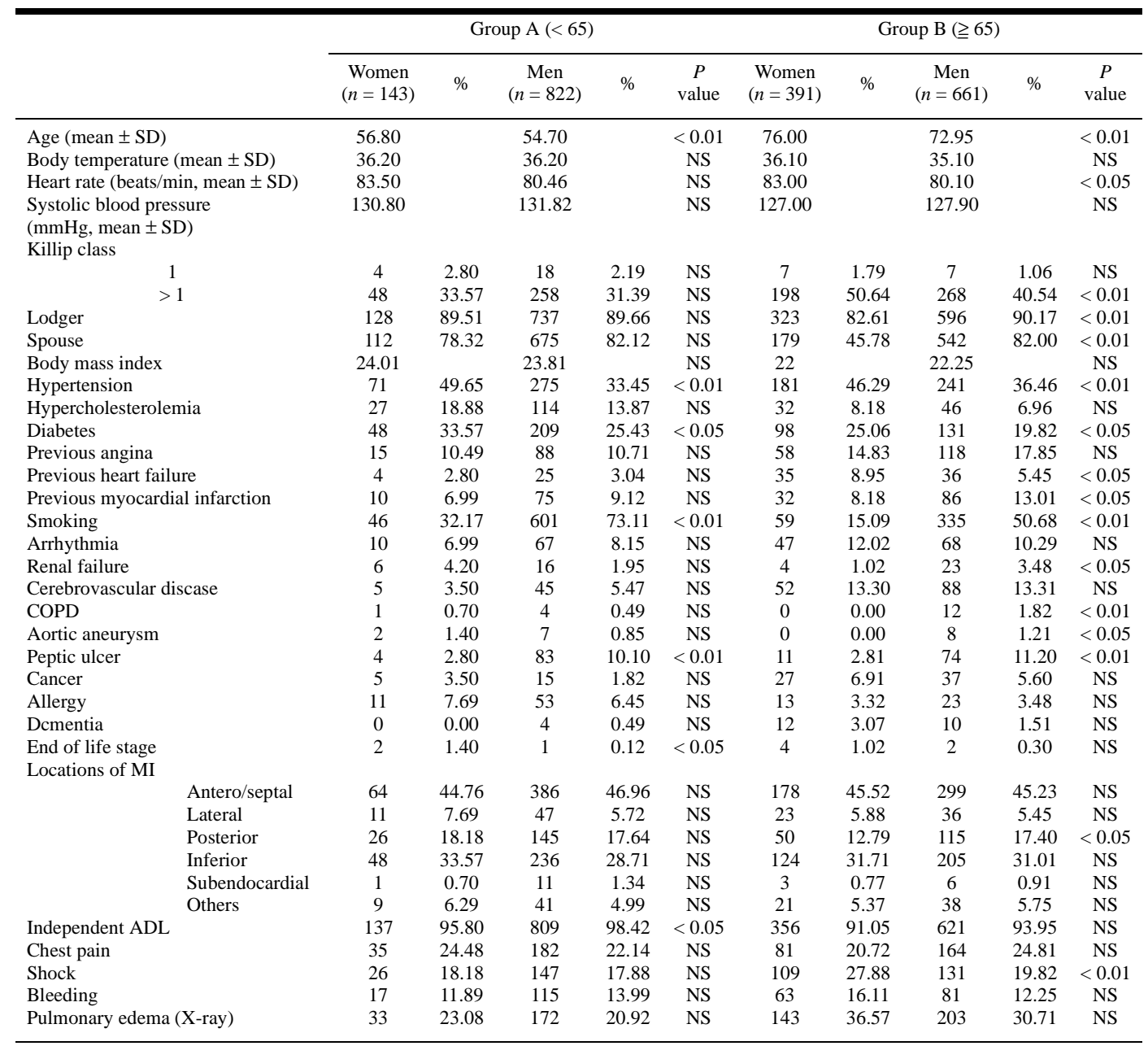

Group A indicates young group; Group B, old group; COPD, chronic obstructive pulmonary disease; MI, myocardial infarction; and ADL, activity of daily living. 
was documented, then the comorbid condition was recorded as absent.

Statistical analysis: We compared the baseline and procedure characteristics and clinical outcomes between women and men according to two age groups $(<65$, 65). Statistical analysis was performed using the chi-square test for categorical variables and the unpaired t test for continuous variables. We also performed multiple logistic regression analysis to identify the independent association between gender and cardiac care according to the age group, after adjusting for other baseline and procedural factors that differed significantly between women and men. Univariate predictors of the cardiac care with a $P$ value less than 0.05 could be used in the model. We present the results as odds ratios (OR) and $95 \%$ confidence intervals $(\mathrm{CI})$. A $P$ value less than 0.05 was considered statistically significant.

\section{RESULTS}

Baseline characteristics: A total of 143 younger women, 822 younger men, 391

Table II. Procedural Characteristics of the Patients

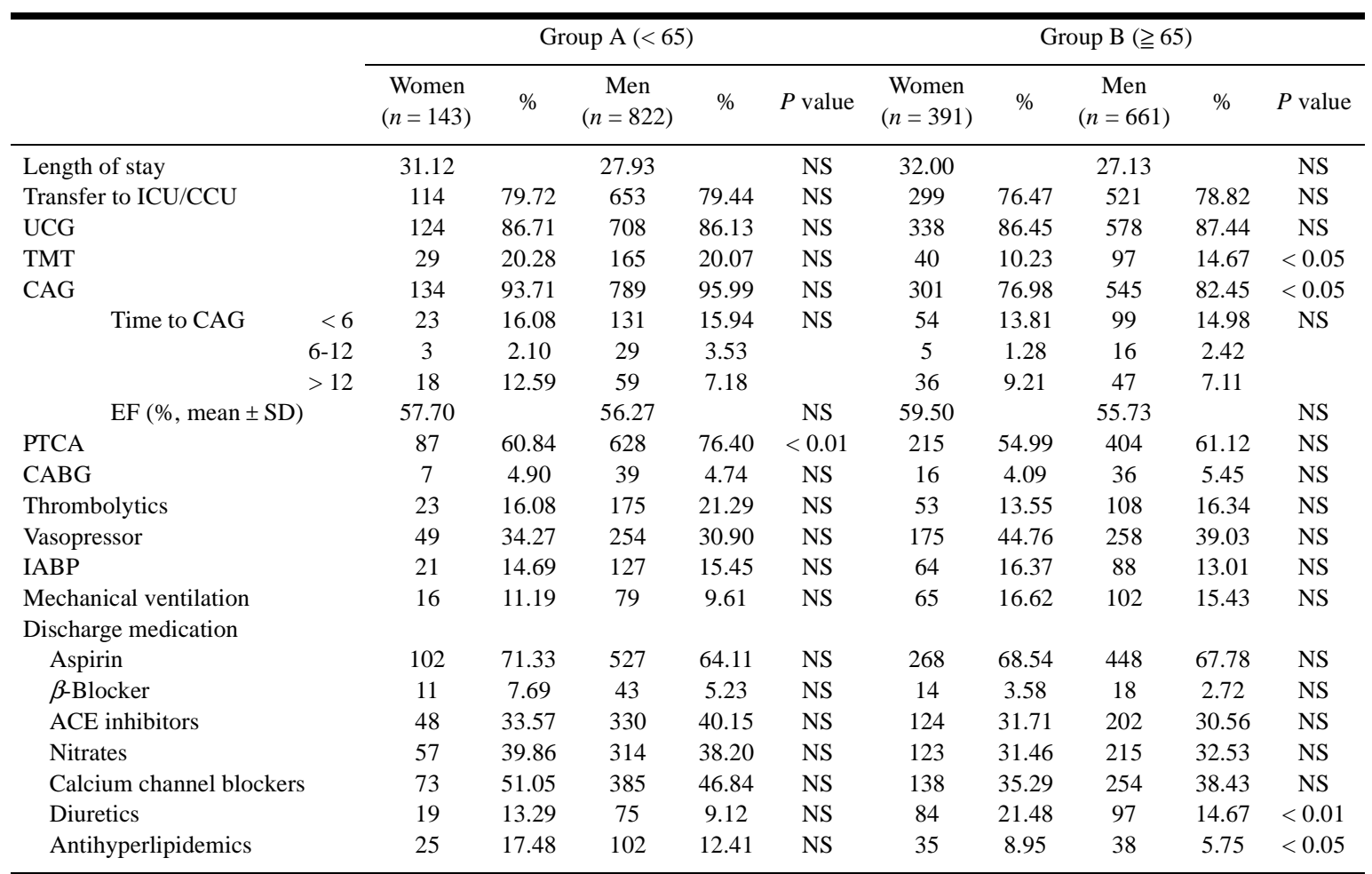

ICU/CCU indicates intensive care unit/coronary care unit; UCG, ultrasound-echocardiogram; TMT, treadmill test; CAG, coronary angiography; EF, ejection fraction; PTCA, percutaneous coronary intervention; CABG, coronary artery bypass graft; and IABP, intraaortic balloon pump. 
older women, and 611 older men were included in the analysis. Among the group under age 65 (the young group), the women were significantly older than the men (Table I). They were more likely to have a history of hypercholesterolemia or diabetes and to be in the end-of-life of all sorts of diseases, but they were less likely to have a history of smoking or peptic ulcer disease. Women were more likely to be independent with respect to their activities of daily living.

Among the elderly, the women were significantly older than the men. The initial heart rate was higher in women. Women were more likely to have signs of heart failure on presentation (Killip class > I, 50.6\% versus $40.5 \%$, respectively). They were more likely to have a history of hypercholesterolemia, diabetes or heart failure, but less likely to have a lodger, a spouse, a history of myocardial infarction, smoking, renal failure, or peptic ulcer disease. Women were more likely to develop cardiogenic shock and less likely to present with posterior infarction.

Procedural characteristics: Among the young, women stayed in hospital almost as long as men (Table II). They were less likely to undergo PTCA. Gender was not associated with the likelihood of receiving TMT or CAG. Among the elderly, women were less likely to receive TMT or CAG (Table IV). They were also less

Table III. Use of Medication, Diagnostic Test and Treatment for AMI Among Young Patients (Below 65) Among Women Versus Men

\begin{tabular}{|c|c|c|c|c|c|c|}
\hline & Unadjusted OR & $95 \% \mathrm{CI}$ & Age adjusted OR & $95 \% \mathrm{CI}$ & $\begin{array}{l}\text { Multivariable } \\
\text { adjusted OR* }\end{array}$ & $95 \% \mathrm{CI}$ \\
\hline Aspirin & 1.39 & $0.94-2.06$ & 1.41 & $0.95-2.08$ & 1.51 & $0.98-2.31$ \\
\hline$\beta$-Blocker & 1.51 & $0.76-3.00$ & 1.64 & $0.82-3.30$ & 1.53 & $0.72-3.28$ \\
\hline ACE inhibitors & 0.75 & $0.52-1.10$ & 0.73 & $0.50-1.07$ & 0.79 & $0.52-1.21$ \\
\hline Lipid lowering therapy & 1.50 & $0.93-2.41$ & 1.61 & $0.99-2.61$ & 1.55 & $0.91-2.61$ \\
\hline Nitroglycerin & 1.07 & $0.75-1.54$ & 1.05 & $0.73-1.52$ & 1.14 & $0.76-1.71$ \\
\hline Ca channel blocker & 1.18 & $0.83-1.69$ & 1.22 & $0.85-1.74$ & 1.05 & $0.71-1.55$ \\
\hline Diuretics & 1.53 & $0.89-2.61$ & 1.46 & $0.85-2.50$ & 1.24 & $0.68-2.25$ \\
\hline Vasopressor & 1.17 & $0.80-1.71$ & 1.09 & $0.74-1.59$ & 1.02 & $0.67-1.55$ \\
\hline Thrombolytics & 0.73 & $0.45-1.18$ & 0.74 & $0.46-1.20$ & 0.75 & $0.44-1.29$ \\
\hline $\mathrm{IABP}$ & 0.91 & $0.55-1.53$ & 0.88 & $0.52-1.48$ & 0.78 & $0.43-1.41$ \\
\hline Mechanical ventilation & 1.16 & $0.65-2.05$ & 1.08 & $0.61-1.92$ & 0.90 & $0.47-1.71$ \\
\hline CAG & 0.59 & $0.27-1.26$ & 0.61 & $0.28-1.31$ & 0.60 & $0.25-1.46$ \\
\hline PTCA & 0.49 & $0.33-0.71$ & 0.51 & $0.35-0.74$ & 0.54 & $0.35-0.82$ \\
\hline CABG & 1.03 & $0.45-2.34$ & 0.95 & $0.41-2.18$ & 0.85 & $0.35-2.08$ \\
\hline UCG & 1.00 & $0.55-1.82$ & 0.98 & $0.53-1.78$ & 0.89 & $0.46-1.75$ \\
\hline TMT & 1.03 & $0.66-1.60$ & 1.03 & $1.02-1.08$ & 1.15 & $0.70-1.89$ \\
\hline
\end{tabular}

AMI indicates acute myocardial infarction; UCG, ultrasound-echocardiogram; TMT, treadmill test; CAG, coronary angiography; PTCA, percutaneous coronary intervention; CABG, coronary artery bypass graft; IABP, intra-aortic balloon pump; OR, odds ratio; and CI, confidence interval.

*Controlling for age, hypertension, diabetes, smoking, peptic ulcer, end-of life stage, and independence of activity of daily living. 
Table IV. Use of Medication, Diagnostic Test and Treatment for AMI Among Old Patients (65 and Older) Among Women Versus Men

\begin{tabular}{|c|c|c|c|c|c|c|}
\hline & Unadjusted OR & $95 \% \mathrm{CI}$ & Age adjusted OR & $95 \% \mathrm{CI}$ & $\begin{array}{l}\text { Multivariable } \\
\text { adjusted OR* }\end{array}$ & $95 \% \mathrm{CI}$ \\
\hline Aspirin & 1.04 & $0.80-1.36$ & 1.03 & $0.78-1.35$ & 0.92 & $0.65-1.31$ \\
\hline$\beta$-Blocker & 1.41 & $0.69-2.89$ & 1.59 & $0.76-3.33$ & 1.34 & $0.51-3.53$ \\
\hline ACE inhibitors & 1.06 & $0.81-1.39$ & 1.18 & $0.89-1.56$ & 1.20 & $0.83-1.74$ \\
\hline Lipid lowering therapy & 1.61 & $1.00-2.60$ & 2.18 & $1.33-3.57$ & 2.79 & $1.47-5.28$ \\
\hline Nitroglycerin & 0.96 & $0.73-1.25$ & 0.99 & $0.76-1.31$ & 1.22 & $0.85-1.77$ \\
\hline Ca channel blocker & 0.88 & $0.68-1.14$ & 1.00 & $0.77-1.31$ & 0.94 & $0.66-1.35$ \\
\hline Diuretics & 1.59 & $1.15-2.20$ & 1.44 & $1.04-2.01$ & 1.05 & $0.68-1.62$ \\
\hline Vasopressor & 1.24 & $0.96-1.60$ & 1.14 & $0.88-1.48$ & 0.84 & $0.56-1.25$ \\
\hline Thrombolytics & 0.78 & $0.54-1.11$ & 0.86 & $0.59-1.24$ & 0.96 & $0.60-1.55$ \\
\hline IABP & 1.24 & $0.86-1.77$ & 1.42 & $0.98-2.05$ & 1.21 & $0.71-2.06$ \\
\hline Mechanical ventilation & 1.06 & $0.76-1.50$ & 1.10 & $0.78-1.57$ & 0.70 & $0.41-1.20$ \\
\hline CAG & 0.69 & $0.51-0.95$ & 1.10 & $0.77-1.55$ & 1.35 & $0.83-2.20$ \\
\hline PTCA & 0.74 & $0.58-0.96$ & 0.93 & $0.71-1.22$ & 1.25 & $0.87-1.80$ \\
\hline $\mathrm{CABG}$ & 0.73 & $0.4-1.34$ & 0.87 & $0.47-1.60$ & 0.68 & $0.28-1.64$ \\
\hline UCG & 0.69 & $0.44-1.06$ & 0.70 & 0.44-1.09 & 0.79 & $0.47-1.33$ \\
\hline TMT & 0.65 & $0.44-0.96$ & 0.76 & $0.51-1.14$ & 0.89 & $0.51-1.58$ \\
\hline
\end{tabular}

See Table III for abbreviations.

*Controlling for age, heart rate, Killip class, spouse, hypertension, diabetes, previous heart failure, previous myocardial infarction, smoking, renal failure, chronic obstructive pulmonary disease, aortic aneurysm, peptic ulcer, AMI location, and cardiogenic shock.

likely to receive PTCA, but not significantly. Women were more likely to receive therapy with diuretics or antihyperlipidemics during their hospital stay.

Multivariable analyses: Multiple regression analysis was carried out to more systemically examine the relations between gender and in-hospital management while controlling for differences in baseline variables, in which statistically significant differences were detected between women and men. Because we considered the presence of a lodger to be strongly correlated to the presence of a spouse, the presence of a lodger was not included in our regression models. The multivariable-adjusted results of in-hospital management are shown in Tables III and IV. Among the young, after controlling for these baseline variables, women were significantly less likely to receive PTCA than men (OR, 0.54, 95\%CI, 0.35-0.82) (Table III). Among the elderly, women were likely to receive therapy with diuretics or antihyperlipidemics, and less likely to receive CAG, PTCA, or TMT before controlling for differences in the previously described variables (Table IV). After controlling, the differences disappeared, and only lipid-lowering therapy tended to be more frequent in women than in men (OR, 2.79, 95\% CI, 1.47-2.58). 


\section{Discussion}

This large-scale retrospective study examined the influence of age and gender in Japan on the delivery of cardiac management in patients with acute myocardial infarction. We expected, on the basis of previous studies, ${ }^{2,9)}$ that differences in in-hospital management between women and men with AMI would be influenced by age, and divided the study subjects into two different age groups. In fact, our results suggested that there were age-specific gender differences in the in-hospital management of patients hospitalized with AMI in the Tokai region of Japan. Namely, our results indicated that lipid-lowering medications were used more frequently among elderly women, and that PTCAs were used less frequently among younger women. It was previously reported that women were more likely to receive lipid-lowering medications ${ }^{13)}$ and less likely to undergo PTCA ${ }^{1,11)}$ than men. Our results were also in accordance with those of previous studies, which have shown that women and men were different in their baseline backgrounds; ${ }^{1,3,16)}$ women are older, and more likely to have hypertension, diabetes mellitus, or previous heart failure, while men are more likely to have had a history of smoking or previous myocardial infarction.

With regards to lipid-lowering therapy, our findings suggested that older women were more likely to receive lipid-lowering therapy even after multivariable adjustment. Some previous studies have reported similar results as ours; ${ }^{13)}$ however, Bakler, et al suggested that women were less likely to receive lipid-lowering therapy. ${ }^{1)}$ It is still unclear whether or not a difference in the incidence of lipid-lowering therapy between women and men exists. In addition, the reason why women were treated more with lipid-lowering therapy is unclear. However, the following explanation may be a good one as to why there is an overuse of lipid-lowering therapy among elderly women: compared to men, the number of women with hyperlipidemia increases dramatically after menopause. In addition, Yarzebski, et al pointed out that less women than men were having their serum cholesterol level measured. ${ }^{18)}$ Therefore, it is conceivable that older women were more likely to have been newly diagnosed with hyperlipidemia during their hospitalization, at which point they started receiving lipid-lowering therapy. In addition, it is possible that older women may be more shocked than older men upon learning they have high cholesterol levels, and that because of this, they request lipid-lowering therapy. Because there is, to our knowledge, no study to support this conjecture, additional study is needed to support the hypothesis that elderly women are shocked more frequently. Among the elderly only, the results of our study suggest that women are more likely to receive a diuretic, as some previous studies have suggested. ${ }^{1,16)}$ However, the difference in the incidence of diuretic treatment disappeared after adjustment for multivariables, including shock and 
the Killip class, which were representative of the severity of the heart failure. This suggests that the more frequent occurrence of severe heart failure among women caused the more frequent administration of diuretics in women. ${ }^{16)}$

Our results do not suggest that women were less likely to undergo ECG or TMT. In addition, they also suggested that TMT was not performed frequently. Leslie, et al demonstrated that women may be less likely to undergo TMT, because this test, compared to CAG, cannot sufficiently predict ischemic events in women. ${ }^{2)}$ The underuse of TMT may no longer be problematic.

In our study, there were no gender differences in the incidences of CAG and $\mathrm{CABG}$ in both age groups after age and multivariable adjustment. However, with regards to PTCA, even after multivariable adjustment, women had undergone less PTCA than men in the younger population, though not in the older one. Because the rate of coronary angiography and time to coronary angiography is similar between young men and young women, the lower use of PTCA may be due to a higher prevalence of insignificant coronary stenoses in women. ${ }^{19,20)}$ Other reasons which may account for why women underwent less PTCA include failure on the part of the physician to offer it, or reluctance on the part of the women to consent to their physician's recommendations. Some researchers have suggested the following as concrete reasons:

- Female patients' lack of knowledge of AMI symptoms may cause a delay in their seeking medical assistance for those symptoms. ${ }^{21)}$

- Gender of physician - a male physician tends to treat a female patient less aggressively. ${ }^{22)}$

- Gender difference in AMI symptoms - females complain of AMI symptoms such as chest pain even when AMI is absent. ${ }^{17,19,23-25)}$

- Gender bias - women or minorities tend to be treated less aggressively by physicians. $^{5,26)}$

- Gender difference in socioeconomic status - women may have a lower economic status, and are more likely to hesitate undergoing an expensive treatment such as PTCA. ${ }^{12,19)}$

Further studies are needed to prove these hypotheses.

Study limitations: There are several important limitations to this study. First, the sample size was not as large as that used in previous studies. Databases for the study of AMI in Japan are not available. Therefore, we had to build a database for our study by ourselves, by visiting the participating hospitals. However, the present study is valuable and contains many interesting findings. Presently, there is no national database in non-Western countries which can be compared with Western countries. ${ }^{3)}$ As mentioned above, some researchers have suggested that gender differences in AMI management may be caused by socioeconomic factors. Our results may be highly suggestive of this. 
Second, we began collecting data in 2001, and spent over three years completing this data set. Therefore, it may have become too old to analyze. It is timeconsuming to conduct chart reviews, and it is ethically problematic to have many examiners coming into hospitals and obtaining personal data, although we did obtain approval from the ethical committee at each hospital. We have also been conducting a prospective study (TAMIS-II) since 2001. TAMIS-II is investigating the same issues as TAMIS-I. We will be able to show temporal trends in AMI management by analyzing the results of TAMIS-II.

Third, our database does not always capture the full extent of the characteristics and clinical courses of the study subjects. Some physicians in the participating hospitals collected data together in their own hospitals; however, trained nurses to whom we gave a lecture on cardiovascular disease collected most of our dataset. Due to the lack of examiners, we should have secured personnel to regularly collect data. Although we trained the nurses and made sure that they could collect the data in practice, it is possible that the data collecting procedures varied among the hospitals depending on who was in charge of the data collection in each hospital, because not all trained nurses are familiar with medical data on circulatory illnesses.

Finally, although our intention was to construct a comprehensive questionnaire, there was a lot of missing laboratory data, such as the distribution of cholesterol levels, CK-MB, or ST elevation.

Conclusion: We conducted a retrospective study to investigate the age differences in the delivery of cardiac management to women versus men with acute myocardial infarction in Japan. The results suggest that there were age-specific gender differences in the in-hospital management of patients hospitalized with AMI. Younger women with AMI are less likely than younger men to receive PTCA. Older women with AMI are more likely to receive lipid-lowering therapy. Our study was limited due to the fact that the data collected was old and because of the small sample size. We intend to analyze our prospective study (TAMIS-II) to confirm the results obtained herein.

\section{REFERENCES}

1. Bakler T, Baburin A, Teesalu R, Rahu M. Comparison of management and 30-day mortality of acute myocardial infarction in men versus women in Estonia. Acta Cardiol 2004; 59: 275-81.

2. Harrold LR, Esteban J, Lessard D, et al. Narrowing gender differences in procedure use for acute myocardial infarction: insights from the Worcester heart attack study. J Gen Intern Med 2003; 18: 423-31.

3. Zubaid M, Rashed WA, Thalib L, Suresh CG. Differences in thrombolytic treatment and in-hospital mortality between women and men after acute myocardial infarction. Jpn Heart J 2001; 42: 669-76.

4. Jelinski SE, Ghali WA, Parsons GA, Maxwell CJ. Absence of sex differences in pharmacotherapy for acute myocardial infarction. Can J Cardiol 2004; 20: 899-905. 
5. Watson RE, Stein AD, Dwamena FC, et al. Do race and gender influence the use of invasive procedures? J Gen Intern Med 2001; 16: 227-34.

6. Herlitz J, Brorsson B, Werko L. Factors associated with the use of various medications amongst patients with severe coronary artery disease. SECOR/SBU Project Group. J Intern Med 1999; 245: 143-53.

7. Kotamaki M, Strandberg TE, Nieminen MS. Clinical findings, outcome and treatment in patients $>$ or $=75$ years with acute myocardial infarction. Eur J Epidemiol 2003; 18: 781-6.

8. Bertoni AG, Bonds DE, Lovato J, Goff DC, Brancati FL. Sex disparities in procedure use for acute myocardial infarction in the United States, 1995 to 2001. Am Heart J 2004; 147: 1054-60.

9. Harrold LR, Lessard D, Yarzebski J, Gurwitz JH, Gore JM, Goldberg RJ. Age and sex differences in the treatment of patients with initial acute myocardial infarction: a community-wide perspective. Cardiology 2003; 99: $39-46$.

10. Oe K, Shimizu M, Ino H, et al. Effects of gender on the number of diseased vessels and clinical outcome in Japanese patients with acute coronary syndrome. Circ J 2002; 66: 435-40.

11. Rathore SS, Foody JM, Radford MJ, Krumholz HM. Sex differences in use of coronary revascularization in elderly patients after acute myocardial infarction: a tale of two therapies. Chest 2003; 124: 2079-86.

12. Karciogcaron;lu O, Unal Aslan B, Aslan O. Gender differences in the management and survival of patients with acute myocardial infarction. Eur J Intern Med 2002; 13: 474-9.

13. McCormick D, Gurwitz JH, Lessard D, Yarzebski J, Gore JM, Goldberg RJ. Use of aspirin, $\beta$-blockers, and lipid-lowering medications before recurrent acute myocardial infarction: missed opportunities for prevention? Arch Intern Med 1999; 159: 561-7.

14. Woon VC, Lim KH. Acute myocardial infarction in the elderly- the differences compared with the young. Singapore Med J 2003; 44: 414-8.

15. Montague T, Montague P, Barnes M, et al. Acute myocardial infarction in Canada: new epidemiologic insights on incidence, therapy, and risk. J Thromb Thrombolysis 1996; 3: 101-5.

16. Herlitz J, Bang A, Hartford M, Karlson BW. Influence of gender on survival, mode of death, reinfarction, use of medication, and aspects of well being during a period of five years after onset of acute myocardial infarction. Clin Cardiol 1996; 19: 555-61.

17. Vaccarino V. Women and outcomes of coronary artery bypass surgery: do we have an answer? Am Heart $\mathbf{J}$ 2003; 146: 935-7.

18. Yarzebski J, Spencer F, Goldberg RJ, Lessard D, Gore JM. Temporal trends (1986-1997) in cholesterol level assessment and management practices in patients with acute myocardial infarction: a population-based perspective. Arch Intern Med 2001; 161: 1521-8.

19. Hochman JS, Tamis JE, Thompson TD, et al. Sex, clinical presentation, and outcome in patients with acute coronary syndrome. Global Use of Strategies to Open Occluded Coronary Arteries in Acute Coronary Syndromes IIb Investigators. N Engl J Med 1999; 341: 226-32.

20. Hochman JS, McCabe CH, Stone PH, et al. Outcome and profile of women and men presenting with acute coronary syndrome: a report from TIMI IIIB. TIMI Investigators. Thrombolysis in Myocardial Infarction. J Am Coll Cardiol 1997; 30: 141-8.

21. Tullmann DF. Gender differences in knowledge of heart attack symptoms in older adults at risk for acute myocardial infarction. Kitasato Int J Nurs Sci 2003; 5: 12-23.

22. Weisse CS, Sorum PC, Sanders KN, Syat BL. Do gender and race affect decisions about pain management? J Gen Intern Med 2001; 16: 211-7.

23. Moser DK, Dracup K, McKinley S, et al. An international perspective on gender differences in anxiety early after acute myocardial infarction. Psychosom Med 2003; 65: 511-6.

24. Patel H, Rosengren A, Ekman I. Symptoms in acute coronary syndromes: does sex make a difference? Am Heart J 2004; 148: 27-33.

25. Milner KA, Vaccarino V, Arnold AL, Funk M, Goldberg RJ. Gender and age differences in chief complaints of acute myocardial infarction (Worcester Heart Attack Study). Am J Cardiol 2004; 93: 606-8.

26. Antoniucci D, Migliorini A, Moschi G, et al. Does gender affect the clinical outcome of patients with acute myocardial infarction complicated by cardiogenic shock who undergo percutaneous coronary intervention? Cathet Cardiovasc Interv 2003; 59: 423-8. 